\title{
Message Framing for Health: Moderation by Perceived Susceptibility and Motivational Orientation in a Diverse Sample of Americans
}

\author{
John A. Updegraff, \\ Department of Psychology, Kent State University \\ Cameron Brick, \\ Department of Psychological and Brain Sciences, University of California, Santa Barbara \\ Amber S. Emanuel, \\ Department of Community Dentistry and Behavioral Science, University of Florida \\ Roy E. Mintzer, and \\ Herman Ostrow School of Dentistry of the University of Southern California \\ David K. Sherman \\ Department of Psychological and Brain Sciences, University of California, Santa Barbara
}

\begin{abstract}
Objective-The present study examined how gain- and loss-framed informational videos about oral health influence self-reported flossing behavior over a 6-month period, as well as the roles of perceived susceptibility to oral health problems and approach/avoidance motivational orientation in moderating these effects.
\end{abstract}

Method-An age and ethnically diverse sample of 855 American adults were randomized to receive no health message, or either a gain-framed or loss-framed video presented on the Internet. Self-reported flossing was assessed longitudinally at 2 and 6 months.

Results-Among the entire sample, susceptibility interacted with frame to predict flossing. Participants who watched a video where the frame (gain/loss) matched perceived susceptibility (low/high) had significantly greater likelihood of flossing at recommended levels at the 6-month follow-up, compared with those who viewed a mismatched video or no video at all. However, young adults (18-24) showed stronger moderation by motivational orientation than by perceived susceptibility, in line with previous work largely conducted with young adult samples.

Conclusion-Brief informational interventions can influence long-term health behavior, particularly when the gain- or loss-frame of the information matches the recipient's beliefs about their health outcome risks.

Correspondence concerning this article should be addressed to John A. Updegraff, Department of Psychology, Kent State University, Kent, OH 44242-0001. jupdegr1@ kent.edu.

Supplemental materials: http://dx.doi.org/10.1037/hea0000101.supp 


\section{Keywords}

perceived risk; message framing; oral care; oral health; persuasive communication

Health behavior interventions often seek to educate people about the consequences of a health behavior. Information about consequences can be framed as either gains or losses. Gain-framed messages emphasize the benefits of adherence, such as "Flossing daily can lower your risk for periodontitis." Loss-framed messages, on the other hand, emphasize the costs of nonadherence such as "Not flossing daily can increase your risk for periodontitis." Although no overall advantage of gain- versus loss-framed messages exists in many domains of health behavior including oral health (for meta-analysis, see Gallagher \& Updegraff, 2012), the relative effectiveness of gain- and loss-framed health messages depends on characteristics of the message recipient. In this study, conducted over 6 months among a large, age and ethnically diverse sample of Americans, we examine two classes of psychological characteristics - the motivational orientation of the message recipient and beliefs about one's health risk- that have each received considerable support as moderators of successful message framing (Rothman \& Updegraff, 2010).

\section{Motivational Orientation}

People differ in the degree to which they monitor for and respond to favorable versus unfavorable outcomes (Carver \& White, 1994; Elliot \& Thrash, 2002). Approach/avoidance theorists (Carver \& White, 1994; Elliot \& Thrash, 2002) view this disposition as the extent to which people are motivated to approach favorable outcomes (approach orientation) or avoid unfavorable outcomes (avoidance orientation). Regulatory focus theorists (Higgins, 1997) view this disposition as the extent to which people are sensitive to the presence or absence of positive events (promotion regulatory focus) or to the presence or absence of negative events (prevention regulatory focus). Both views converge on the prediction that approach-oriented and promotion-focused people should respond more favorably to gainframed appeals, whereas avoidance-oriented and prevention-focused people should respond more favorably to loss-framed appeals, because congruently framed messages yield more regulatory fit (Lee \& Aaker, 2004) and lead to greater elaboration (Updegraff, Sherman, Luyster \& Mann, 2007). ${ }^{1}$

Support for these predictions was first shown in the domain of oral health (Mann, Sherman, \& Updegraff, 2004; Sherman, Mann, \& Updegraff, 2006; for a review, see Sherman, Updegraff, \& Mann, 2008). Similar effects of matching message frame to motivational orientation have now been observed for HPV vaccination (Gerend \& Shepherd, 2007; Nan, 2012), physical activity (Latimer, Rivers, et al., 2008), calcium consumption (Gerend \& Shepherd, 2013), fruit and vegetable consumption (Latimer, Williams-Piehota, et al., 2008), and smoking prevention (Zhao \& Pechmann, 2007). With few exceptions (Latimer, Rivers, et al., 2008; Latimer, Williams-Piehota, et al., 2008), moderation by motivational orientation

\footnotetext{
${ }^{1}$ Regulatory focus theory makes further distinctions, including (a) promotion-oriented people should be more responsive to gains representing the presence of positive outcomes rather than the absence of negative outcomes and (b) prevention-oriented people should be more responsive to losses representing the presence of negative outcomes rather than the absence of positive outcomes.
} 
has only been shown in adolescent or young adult populations. Meta-analyses show the health behavior of younger people to be driven by different factors than that of middle-aged and older adults (e.g., Albarracin, Johnson, Fishbein, \& Muellerleile, 2001; Hagger, Chatzisarantis, \& Biddle, 2002). Adolescents' physical activity, for example, is more strongly related to personality traits such as achievement orientation and sensation-seeking than it is to specific beliefs about the behavior such as attitudes, outcome expectations, or perceived benefits of physical activity (Sallis, Prochaska, \& Taylor, 2000; see Ulleberg \& Rundmo, 2003, for similar patterns in the domain of adolescent risky driving behavior). Young adults typically have fewer health problems than their older counterparts, and unrealistic optimism about health risks (cf. Renner, Knoll, \& Schwarzer, 2000; Weinstein, 1984). As such, young adults' beliefs about their health status may be less dominant in shaping their responses to health communications than their dispositional motivations.

\section{Perceived Susceptibility}

Perceived susceptibility is another key moderator of people's responses to framed health messages. When people perceive themselves as susceptible to a health condition, they may be especially vigilant against possibility of negative health outcomes; in regulatory focus terms, they are prevention-focused concerning the health issue. In contrast, when people perceive low susceptibility to a health condition, they may be especially eager to achieve and maintain positive outcomes; they are promotion-focused concerning the health issue. This regulatory focus-based account (cf. Lee \& Aaker, 2004) predicts that gain-framed messages should be more effective for those who perceive low susceptibility to a health problem, but loss-framed messages should be more effective for those who perceive high susceptibility.

In a study that manipulated young adults' perceptions of risk for mononucleosis, Lee and Aaker (2004) found that a gain-framed message promoted more interest in a preventive supplement against mononucleosis than a loss-framed message among people told that they were at low risk; in contrast, a loss-framed message promoted more interest for those told that they were at high risk. Naturally occurring beliefs about susceptibility likewise moderate message framing effects, as shown in studies of middle-to-older-aged women considering mammography screening (Gallagher, Updegraff, Rothman, \& Sims, 2011), middle-aged adults considering colorectal cancer screening (Ferrer, Klein, Zajac, Land, \& Ling, 2012), and young adults considering HIV-testing (Hull, 2012). Hull (2012) also showed that perceived susceptibility interacted with message framing to increase participants' elaboration of the message, which mediated the effects of framing on intentions to get tested.

Most studies that have reported a moderating role of people's beliefs about susceptibility have found it among middle- to older-adult samples (Ferrer et al., 2012; Gallagher et al., 2011), perhaps because beliefs about susceptibility to health issues become more salient with age. In the domain of oral health, most message framing studies have utilized adolescent or young adult samples (Mann et al., 2004; Sherman et al., 2006; Uskul, Sherman, \& Fitzgibbon, 2009), perhaps limiting the ability to identify beliefs about 
susceptibility as moderators of message framing effects, although that it should be noted that these aforementioned studies did not assess susceptibility so it remains an open question.

\section{Study Objectives}

Dental caries, gingivitis, and periodontitis are widespread health problems, and may increase one's risk for serious medical conditions such as cardiovascular disease (Beck \& Offenbacher, 2005; Loos, Craandijk, Hoek, Wertheim-van Dillen, \& van der Velden, 2000). Many Americans remain nonadherent to practices that can prevent the onset of oral health problems (McCaul, Glasgow, \& Gustafson, 1985), with over 70\% of American adults not flossing daily and over $30 \%$ not flossing at all (CDC/NCHS, 2010). To address this issue, we evaluated an online message framing intervention to improve oral health behaviors.

We recruited a large sample of Americans, diverse in both age and ethnic/racial background, randomly assigned participants to view an Internet-administered gain- or loss-framed educational video about the importance of dental flossing (or no video), and observed the impact of these videos on flossing over 6 months. We predicted that gain-framed messages would be more persuasive for approach-oriented and low-susceptibility people but lossframed messages would be more persuasive for avoidance-oriented and high-susceptibility people. We also expected that motivational orientation would moderate effects among the young adults in our sample, but expected that perceived susceptibility might moderate among a more age-diverse sample. The no message control condition allowed us to examine whether "mismatched" messages provided any benefit compared with no message at all. When behavioral effects were observed, a secondary aim was to explore potential psychological mediators, including attitudes and intentions.

\section{Method}

\section{Participants}

Participants were recruited by Knowledge Networks ${ }^{\circledR}$, a survey research firm that maintains a large panel of Americans. Details of this panel can be found here: http:// www.knowledgenetworks.com/knpanel/. Knowledge Networks utilizes probability-based sampling with a mix of random-digit-dialing and address-based-sampling to recruit and select members into the panel. Households that have a home computer and Internet access earn incentive points (redeemable for cash) for completing surveys. Households that do not have a computer and access to the Internet are provided with free monthly Internet access and an Internet-enabled device (either laptop or WebTV) in return for completing a short survey weekly. The typical survey commitment for panel members is one survey per week or four per month with a duration of 10 to 15 min per survey, and typical payment is approximately $\$ 5$ per month. Panel members have the option to not respond to any surveys or individual survey items. Participants in this study were drawn from panel members who preferred to complete surveys in English and were invited via an e-mail that did not immediately reveal the focus on oral health until the informed consent process. The inclusion criteria were flossing less than twice a day at baseline, staying on the assigned video page for the full duration of the video ( $>5 \mathrm{~min}$ ), and providing at least one follow-up (see Figure 1 for CONSORT Diagram; Moher, Schulz, \& Altman, 2001). 
Eight-hundred fifty-five participants met inclusion criteria. The sample was $46.6 \%$ female, with average age of 45 , ranging from 18 to 89 . The sample was comparably distributed between Hispanic (28.7\%), Black/African American (26.8\%), White (22.3\%), and Asian (22.2\%) participants. $^{2}$ Due to panel limitations, a sizable proportion of American Indians, Alaska Natives, and Native Hawaiians could not be recruited, although a few participants who identified as Hispanic also identified as Native American or Alaska Native $(N=5)$ or as Native Hawaiian $(N=1)$. Thirty-two percent had a high school education or less, $29.1 \%$ had some college, and $39.0 \%$ had a bachelor's degree or higher. Median household income was approximately $\$ 50,000$ per year.

\section{Procedure}

All surveys were presented online. All participants completed baseline measures of oral health behaviors, perceived susceptibility, and motivational orientation. Participants were then randomly assigned to a gain-framed video $(n=352)$, loss-framed video $(n=334)$, or no video control condition ( $n=169$ ), with an a priori decision to run approximately half as many in the control condition as in each of the framed conditions. Next, participants reported intentions to engage in oral health behaviors. After 2 and 6 months, individuals were again e-mailed (and phoned, if needed) to complete an online follow-up survey about their recent oral health behaviors. Participants who remained at the 6-month survey were no different from those who dropped out on any demographic or health variable including flossing behavior at 2 months, all $p \mathrm{~s}>.20$. However, there was more dropout in the no video control condition than in the video conditions at 2 months $\left(\chi^{2}(1)=3.11, p=.08\right)$ and 6 months $\left(\chi^{2}(1)=7.79, p<.01\right)$. Importantly, there was no significant difference in dropout between the gain- and loss-framed conditions at either 2 months $(p=.85)$ or 6 months ( $p=$. 73).

\section{Measures and Materials}

Past flossing-Frequency of flossing at baseline was assessed with a single item used in a prior national survey of oral health behavior (Davidson, Rams, \& Andersen, 1997): "In general, how often do you floss your teeth?" with response options of never, once per month, a few (2-3) times per month, once per week, a few (2-6) times per week, once per day, and two or more times per day.

Motivational orientation-Motivational orientation was assessed using the Behavioral Inhibition System (BIS) and Behavioral Activation System (BAS) scales (Carver \& White, 1994), which include 13 items that assess approach (BAS) orientation $(\alpha=.86)$ and seven items that assess avoidance (BIS) orientation $(a=.73)$. Consistent with procedures used elsewhere (Mann et al., 2004; Sherman et al., 2006), we computed a measure of predominant motivational orientation by subtracting the mean score on the avoidance items from the mean score on the approach items, so higher values represent stronger approach

\footnotetext{
${ }^{2} \mathrm{~A}$ secondary purpose of recruiting equal numbers of Whites, Blacks, Asians, and Hispanics was to examine whether cultural exposure moderates differential effectiveness of gain versus loss frames (e.g., Uskul et al., 2009). This is the focus of a forthcoming report (Sherman et al., 2014).
} 
relative to avoidance orientation. The BIS/BAS has been validated in adult community samples (Jorm et al., 1998).

Perceived susceptibility-Perceived susceptibility to oral health problems was assessed by a five-item scale (Batchelor \& Sheiham, 2002) on the perceived likelihood of having a filling, getting gum disease, or having other oral health problems within the next 12 months and the next 5 years $(\alpha=.86)$.

Framed videos-Each of the videos were created for this study, were $51 / 2 \mathrm{~min}$ in length, and featured a female dentist who described the health consequences of regular brushing, flossing, and dental visits. Most of the video covered the importance of flossing, including a section with the dentist and a patient that instructed viewers how to floss according to American Dental Association recommendations (ADA, 2013; spool method, at least once per day). Approximately $40 \%$ of the script consisted of framed statements. Pilot testing validated the manipulation of framing and showed that the videos were easy to understand and pay attention to (see supplementary materials for more details and video scripts).

Potential mediators-At the end of the baseline survey and the 2-month survey, several possible mediating beliefs were assessed. Intention to floss was assessed by a single item "How many times do you plan to floss your teeth in the upcoming week?" with options ranging from 0-14 (see also Sherman et al., 2006). Attitude toward flossing was assessed with a single item developed for this study that asked "What is the value of flossing your teeth?" $(1=$ extremely worthless, $5=$ extremely valuable $)$. Self-efficacy specifically related to dental flossing was assessed with seven items used in prior research (Sherman et al., 2006), $a=.95$.

Flossing at 2 and 6 months-To obtain a sensitive measure of flossing behavior at each of the follow-ups, we asked participants to report how many times in the past week they flossed their teeth, on a scale ranging from $0-14$. This measure shows significant correlation with clinical indices of oral health (CDC/NCHS, 2010).

\section{Analytic Strategy}

We used generalized estimating equations (GEE; Liang \& Zeger, 1986) in Stata 12 to examine the influence of the framed video messages on flossing across the 6-month study period. GEE is an extension of the generalized linear model for regressions involving repeated outcomes. By analyzing both follow-up waves simultaneously, Type I error is reduced. When a significant effect was found across follow-ups, we used multiple regression to identify the influence of framing separately at each of the waves. Missing data other than attrition was negligible (see Figure 1); thus, missing values were not imputed. As our hypotheses predicted interactions between perceived susceptibility, motivational orientation, and message framing, we use procedures (outlined by Aiken \& West, 1991) to test moderation. Continuous measures were mean-centered prior to computing product terms with the message framing variable. To ensure that standard errors and all associated significance tests were robust to any violations of normality in the residuals, we report bootstrapped standard errors. Baseline demographic differences in flossing were also 
examined. Females $(M=4.25, S D=1.73)$ flossed more than males $(M=3.69, S D=1.86), p$ $<.001$. Asians $(M=4.32, S D=1.77)$ flossed significantly more than Caucasians $(M=4.00$, $S D=1.72)$, Hispanics $(M=3.91, S D=1.85)$, and Blacks $(M=3.63, S D=1.86)$ combined, $p$ $=.001$; Blacks flossed significantly less than all other groups combined, $p=.002$. Higher age $(r=.13)$ and income $(r=.16)$ were associated with greater flossing. These variables were retained as covariates in all further analyses.

\section{Results}

\section{Comparison of Video and Control Groups}

Table 1 presents descriptive data by experimental condition. We first examined the effects of our videos on subsequent flossing by comparing those who viewed either of the framed videos with those in the control condition, while controlling for past flossing. Participants who saw a video reported flossing significantly more $(M=3.82, S E=.057)$ than those who did not see a video $(M=3.48, S E=.11), B=.29, S E=.12, p=.017$, Cohen's $d=.15$.

\section{Moderators of Message Framing Effects}

Next we examined the relative effectiveness of the gain- and loss-framed videos and their interaction with perceived susceptibility and motivational orientation (see Table 2 for results of GEE analysis), while also controlling for past flossing and demographics. There was no overall effect of gain versus loss framing $(p=.53)$. However, there was a significant Frame $\times$ Perceived Susceptibility interaction $(p=.03)$, indicating that the influence of message framing on flossing depended on the recipient's level of perceived susceptibility. ${ }^{3}$ For participants with low $(-1 S D)$ perceived susceptibility, those who viewed the gain-framed video flossed significantly more across the 6-month period $(M=3.97, S E=.12)$ than those who viewed the loss-framed video $(M=3.60, S E=.11), z=2.32, p=.02$. For participants with high $(+1 S D)$ perceived susceptibility, this pattern was reversed; those who viewed the loss-framed video flossed somewhat but not significantly more $(M=3.96, S E=.13)$ than those who viewed the gain-framed video $(M=3.75, S E=.13), z=1.22, p=.22$.

The 2- and 6-month follow-ups were separately examined for the Frame $\times$ Susceptibility effect. At 2 months, the Frame $\times$ Susceptibility was not significant. At the 6-month followup, the Frame $\times$ Susceptibility interaction was significant $(p=.004$, see Figure 2$){ }^{4}$ Consistent with the pattern observed in the GEE analyses, among the participants with low perceived susceptibility $(-1 S D)$, those who viewed the gain-framed video flossed more $(M$ $=4.35, S E=.25)$ than those who viewed the loss-framed video $(M=3.44, S E=.25), z=$ $2.56, p=.01$. Among participants at high perceived susceptibility $(+1 S D)$, this pattern was different, with those who viewed the loss-framed video flossing somewhat but not significantly more $(M=4.19, S E=.26)$ than those who viewed the gain-framed video $(M=$ $3.83, S E=.24), z=1.01, p=.31$.

\footnotetext{
${ }^{3}$ This Perceived Risk $\times$ Framing interaction was not moderated by ethnicity.

${ }^{4}$ To ensure that this finding was not attributable to attrition, we also conducted a last-observation-carried-forward analysis in which 2 month flossing values were carried forward to 6-months for participants who dropped out at 6-months. In this analysis, The Frame $\times$ Susceptibility interaction was likewise significant $(B=-.54, p=.014)$.
} 
To examine the potential practical effect of this finding, we examined the proportion of participants flossing once a day or more at the 6-month follow-up as a function of match between frame and perceived susceptibility. People considered as matches $(N=276)$ were either (a) those with susceptibility at or above 3 and who received a loss-framed message, or (b) those with susceptibility below 3 and who received a gain-framed message; mismatches $(N=290)$ were the remaining participants. Indeed, a significant matching effect was observed, as shown in Figure 3. A greater proportion of participants who received a matched message flossed at recommended rates $(34.5 \%, S E=2 \%)$ compared with those with a mismatched message $(27.4 \%, S E=2 \%)$, odds ratio $(\mathrm{OR})=1.58, S E=.35, p=.04$. Incorporating the no video group into analyses, planned contrasts showed that matched participants were more likely to floss at recommended levels than those in the mismatched and no video groups combined ( $\mathrm{OR}=1.50, S E=.29, p=.03$ ), and those in the mismatched group were no more likely to floss at recommended levels than those in the no video group $(30.3 \%, S E=3 \% ; \mathrm{OR}=.80, S E=.21, p=.40)$.

\section{Moderation Among Young Adults}

As Table 2 shows, there was no significant moderation of framing by motivational orientation. Because these results are at odds with past findings of A Frame $\times$ Motivational Orientation interaction found in mostly young adult samples, we ran an additional GEE model (as described earlier) restricted to participants younger than 25 ( $N=62 ; 35$ females), an age cutoff frequently used in meta-analytic reviews (e.g., Hagger et al., 2002; Sheeran \& Orbell, 1998). Within this subsample, the Frame $\times$ Motivational Orientation interaction was significant $(B=2.60, S E=1.20, z=2.16, p=.031)$ but the Frame $\times$ Susceptibility interaction was not significant $(B=-.14, S E=.36, z=-.40, p=.69) .{ }^{5}$ Follow-up regression analyses showed that the Frame $\times$ Motivational Orientation predicted flossing at 6 months $(B=5.17, S E=2.03, z=2.55, p=.011)$ but not at 2 months $(B=.12, S E=1.83, z=.07 . p$ $=.95){ }^{6}$ Among approach-oriented young adults, the gain-framed video led to significantly greater flossing at 6 months $(M=3.04, S E=.56)$ than the loss-framed video $(M=0.26, S E$ $=.12), z=4.99, p<.001$. However, among avoidance-oriented young adults, the lossframed video led to greater flossing $(M=4.34, S E=.92)$ than the gain-framed video $(M=$ $2.29, S E=.43), z=2.13, p=.03$. Thus, young adults in this sample exhibited a pattern of framing effects consistent with prior work using young adults.

\section{Mediation of Behavioral Effects}

Because behavioral effects of framing and perceived susceptibility were observed across the 6-month study period, we also examined whether attitudes, intentions, or self-efficacy might have mediated this effect, assessed immediately after the video and at the 2-month followup. None of these tests were significant, suggesting that the interaction between framing and

\footnotetext{
${ }^{5}$ We also examined the age-dependence of framing effects by including age as a moderator variable using the full sample. A marginally significant Frame $\times$ Motivational Orientation $\times$ Age interaction was found $(p=.086)$, showing that the congruency effect became stronger with decreasing age. In contrast, the Frame $\times$ Susceptibility $\times$ Age interaction was not significant $(p=.31)$, suggesting that it was largely robust across the age spectrum of our sample.

${ }^{6}$ Both BIS and BAS contributed to this interaction. As BIS increased, loss frames became more effective (BIS $\times$ Frame $B=-5.45, p$ $=.04$ ). As BAS increased, gain frames became somewhat more effective (BAS $\times$ Frame $B=4.63, p=.14$ ).
} 
perceived susceptibility was not explained by changes in participants' stated attitudes, intentions, or efficacy regarding flossing.

However, another pathway by which perceived susceptibility and framing might interact to predict flossing is by changing how much a mediator predicts subsequent flossing: in other words, by influencing the "intention-behavior gap" (Sheeran, 2002). To test this possibility, we conducted a regression analysis that predicted flossing at the 6-month follow-up from 2month intentions to floss, framing condition, perceived susceptibility, and their 2- and 3-way interactions, controlling for flossing at the 2-month follow-up. The 3-way interaction was key, as it represented the interaction between intentions and the 2-way Framing $\times$ Susceptibility interaction on subsequent flossing, and it was significant, $B=-.19, S E=.08$, $p=.02$, indicating that the interaction between susceptibility and framing moderated the link between intentions and behavior.

To simplify interpretation, we collapsed the Frame $\times$ Susceptibility interaction into a single variable representing match versus mismatch, as described earlier. Among those who viewed a matched video, intentions significantly predicted subsequent flossing, $B=.26, S E$ $=.05, p<.001$. Among those who viewed a mismatched video, intentions predicted subsequent flossing $(B=.13, S E=.05)$ but to a significantly lesser extent (difference test $t=$ $2.58, p=.01$ ). Thus, the interaction between framing and perceived susceptibility may have influenced long-term flossing not by raising intentions, but by leading to intentions that more reliably predicted behavior.

\section{Discussion}

Consistent with recent approaches to health message framing, we found that the effectiveness of gain- and loss-framed messages depended on individual differences of the message recipient. Most generally, individuals' perceived susceptibility to health problems shaped their responses to framed appeals. This moderation by susceptibility has been found in other studies of screening behaviors (Ferrer et al., 2012; Gallagher et al., 2011; Hull, 2012), but has never extended to preventive behaviors or been demonstrated over as extended of a timeframe as in this study. We showed significantly greater odds of adhering to the American Dental Association's recommended rate of flossing at the 6-month followup among participants who received a brief intervention in which the frame was matched to their beliefs about susceptibility, as compared with those who received an intervention with a mismatched frame or no intervention at all (these groups did not differ significantly). Given that framing is an inevitable part of most health educational interventions, these findings attest to the benefits of strategically tailoring the frame of a health behavior intervention to the relevant health beliefs of the recipient.

\section{Theoretical Implications}

This is the first study to show that naturally occurring beliefs about susceptibility shape people's responses to framed messages in promoting an illness prevention behavior such as dental flossing. Framing effects were most pronounced among participants with low perceived susceptibility, underscoring the importance of using framing as a way to motivate healthy behavior among people who may not yet perceive a risk. Although the pattern of 
framing was somewhat reversed among participants with high perceived susceptibility, it was not significant. Prior studies that have examined the interaction between perceived susceptibility and framing have found the effects either driven by those with low perceived susceptibility (Hull, 2012, HIV intentions; Lee \& Aaker, 2004, impressions of monofighting supplement ads), or by those with high perceived susceptibility (Ferrer et al., 2012, colorectal cancer screening intentions; Gallagher et al., 2011, mammography screening). No study has found significant effects of framing at both low and high levels of susceptibility. Thus, although mounting evidence shows the role of perceived susceptibility in moderating effects of framed messages, more work is needed to predict exactly where along the susceptibility spectrum framing effects emerge. Perhaps differences in how people arrive at perceptions of risk may explain some of this variability (Gallagher et al., 2011). With HIV and oral health, people may come to a clear awareness of their low risk status because they have not engaged in known behavioral risk factors for HIV or because they show no symptoms of oral health problems (e.g., pain, bleeding gums). In contrast, risk factors for breast and colorectal cancer are less controllable and less visible than for HIV or oral health, so even people who report low perceived susceptibility may not be fully convinced of their risk status (Gallagher et al., 2011). There are many other factors that could solidify a person's perceived susceptibility to a given health threat—objective risk feedback, genetic testing, regular engagement in preventive behavior-so future research may benefit from examining the interaction of message framing and perceived susceptibility in contexts that involve any of these factors.

Differential moderation by age-Consistent with prior research (see Sherman et al., 2008 for review), motivational orientation moderated framing effects among young adults but not among the full sample. Furthermore, perceived susceptibility did not moderate framing effects among young adults but did among the full sample. It may be that beliefs about perceived susceptibility to health problems are not particularly grounded beliefs for young adults, as they typically experience few health problems and have unrealistic optimism about health risks (cf. Renner, Knoll, \& Schwarzer, 2000; Weinstein, 1984). In situations where people's beliefs about a health issue are not particularly strong —as may be the case with young adults and oral health — their responses to framed messages may be more influenced by dispositional factors such as approach/avoidance motivation orientation (Rothman, Wlaschin, Bartels, Latimer, \& Salovey, 2008) that lead to a greater depth of processing of strongly constructed health arguments (Updegraff et al., 2007). Thus, tailoring framed messages to approach/avoidance motivations may be a useful strategy for interventions targeting young adults, but may not meet with as much success when targeting older individuals.

Framing and the intention-behavior gap-Like many prior framing studies (see Gallagher \& Updegraff, 2012, for review), we did not find evidence that our framing effects were directly mediated by changes in intentions or attitudes toward flossing. Instead, the Frame $\times$ Susceptibility interaction may have been due to a bridging of the intentionbehavior gap. When people viewed a video that matched their perceived susceptibility to oral health problems, their intentions more reliably predicted behavior than when the video was mismatched. This finding is consistent with research by Hull (2012), who found that the 
interaction between perceived susceptibility and framing on intentions to HIV test was mediated by elaborative processing of the message. When elaboration on a persuasive message is high, attitudes should be more stable over time and more predictive of behavior than when elaboration is low (Briñol \& Petty, 2006). Thus, our findings reinforce the argument that matching the frame of a health message to personal factors such as susceptibility or motivational orientation is a simple technique that increases a recipient's attention and elaboration on a health message.

\section{Health Implications}

These findings have implications for oral health research as well. Relatively few behavioral interventions have shown long-term improvements in oral health care among adults (Watt \& Marinho, 2005; but see Halvari \& Halvari, 2006; Stewart et al., 1991; Tedesco, Keffer, Davis, \& Christersson, 1992 for exceptions). Our Internet-delivered framed videos produced more self-reported flossing behavior over a 6-month period than a no intervention control. Thus, these findings attest to the feasibility of using the Internet in future oral health interventions. Internet-delivered interventions can be less expensive and wider-reaching than intensive, in-person treatments.

The long-term behavior change we observed should be interpreted within the context of the manipulation's brevity: It was just a 5-min educational video. Other oral health interventions have been more intensive, utilizing in-person consultation with dental hygienists over multiple sessions (Halvari \& Halvari, 2006; Stewart et al., 1991; Tedesco et al., 1992). These interventions have shown greater self-reported flossing behavior at 6-month followups compared with no intervention controls, corresponding to effect sizes ranging from small ( $d=.24$ in Tedesco et al., 1992) to large ( $d=1.2$ in Halvari \& Halvari, 2006 and $d=2.4$ in Stewart et al., 1991). In comparison, we found a small but significant effect of our videos on self-reported flossing behavior ( $d=.15$, compared to no video), with the most notable effects emerging when the frame of the video matched people's beliefs about perceived susceptibility ( $d=.19$, compared with no video). Thus, our brief intervention produced effects comparable with one study of an in-person intervention (Tedesco et al., 1992), but not as strong of an effect as interventions that included more than 60 min of interaction with an oral health professional. However, a tailored, Internet-administered intervention can have a far greater reach than in-person oral health interventions. A central challenge for Internetadministered intervention will be getting and holding an audience's attention; we show that matching the frame to beliefs about susceptibility is one strategy that may help. Thus, matching an intervention's frame to beliefs about susceptibility could be profitably incorporated into other types of tailored interventions, whether those interventions be brief or extensive, in-person or online.

\section{Limitations}

There are several limitations of this research that should be noted and addressed in future work. There was considerable attrition in this study. Although there was no difference in attrition between the gain and loss video conditions, there was more attrition in the no video control condition than in the video conditions. Thus, comparisons involving the control condition should be interpreted with some caution. As our study relied on self-reports of 
flossing, future research may benefit by examining the effects of a message framing intervention on more objective measures of behavior or on clinical indices of oral health. Furthermore, attitudes and intentions were assessed with single-item measures. Although we excluded a few participants who skipped through the video early, we could not verify that all participants watched the video; this may have attenuated the influence of the framed videos. Lastly, our study focused on a general population of Americans, but due to panel limitations the study did not include a sizable proportion of American Indians, Alaska Natives, and Native Hawaiians. Future research should evaluate the use of framed interventions in such populations as well as in populations with higher rates of oral health problems both in the U.S. and internationally (see Iranians; Pakpour, Yekaninejad, Sniehotta, Updegraff, \& Dombrowski, in press).

\section{Conclusion}

Framing is an inevitable part of interventions that seek to educate people about the consequences of their health behaviors, and interventions can benefit by strategically using particular message frames. This study integrates and reconciles two bodies of work in the health message framing literature, and yields the following recommendations for the strategic use of framed messages. Among young adults, motivational orientation shapes responses to framed messages. However, among middle- and older-adult samples, <!----> interventions should focus on perceived susceptibility. Future research should also examine the issue of how best to integrate multiple moderators in creating models to examine heterogeneous treatment effects for health psychology interventions. Doing so will build on the present demonstration that long-term changes in American adults' health behavior can be achieved by matching the frame of a single Internet-administered intervention message to people's relevant health beliefs.

\section{Supplementary Material}

Refer to Web version on PubMed Central for supplementary material.

\section{Acknowledgments}

Funding for this project was provided by a grant from the National Institute of Dental and Craniofacial Research (R21 DE019704).

\section{References}

Aiken, LS.; West, SG. Multiple regression: Testing and interpreting interactions. Thousand Oaks, CA: Sage; 1991.

Albarracin D, Johnson BT, Fishbein M, Muellerleile PA. Theories of reasoned action and planned behavior as models of condom use: A meta-analysis. Psychological Bulletin. 2001; 127:142161.10.1037/0033-2909.127.1.142 [PubMed: 11271752]

American Dental Association (ADA). Flossing. 2013. Retrieved from www.mouthhealthy.org/en/aztopics/f/flossing

Batchelor P, Sheiham A. Does perceived risk of oral problems influence the use of dental services in university entrants? Community Dental Health. 2002; 19:116-119. [PubMed: 12146580] 
Beck JD, Offenbacher S. Systemic effects of periodontitis: Epidemiology of periodontal disease and cardiovascular disease. Journal of Periodontology. 2005; 76:2089-2100.10.1902/jop.2005.76.11-S. 2089 [PubMed: 16277581]

Briñol P, Petty RE. Fundamental processes leading to attitude change: Implications for cancer prevention communications. Journal of Communication. 2006; 56:S81-S104.10.1111/j. 1460-2466.2006.00284.x

Carver CS, White TL. Behavioral inhibition, behavioral activation, and affective responses to impending reward and punishment. Journal of Personality and Social Psychology. 1994; 67:319_ 333.10.1037/0022-3514.67.2.319

Centers for Disease Control (CDC) \& National Center for Health Statistics (NCHS). National health and nutrition examination survey data. Hyattsville, MD: CDC; 2010.

Davidson PL, Rams TE, Andersen RM. Socio-behavioral determinants of oral hygiene practices among USA ethnic and age groups. Advances in Dental Research. 1997; 11:245253.10.1177/08959374970110020701 [PubMed: 9549990]

Elliot AJ, Thrash TM. Approach-avoidance motivation in personality. Journal of Personality and Social Psychology. 2002; 82:804-818.10.1037/0022-3514.82.5.804 [PubMed: 12003479]

Ferrer RA, Klein WMP, Zajac LE, Land SR, Ling BS. An affective booster moderates the effect of gain- and loss-framed messages on behavioral intentions for colorectal cancer screening. Journal of Behavioral Medicine. 2012; 35:452-461.10.1007/s10865-011-9371-3 [PubMed: 21850516]

Gallagher KM, Updegraff JA. Health message framing effects on attitudes, intentions, and behavior: A meta-analytic review. Annals of Behavioral Medicine. 2012; 43:101-116.10.1007/ s12160-011-9308-7 [PubMed: 21993844]

Gallagher KM, Updegraff JA, Rothman AJ, Sims L. Perceived susceptibility to breast cancer moderates the effect of gain-and loss-framed messages on use of screening mammography. Health Psychology. 2011; 30:145-152.10.1037/a0022264 [PubMed: 21401248]

Gerend MA, Shepherd M. Message framing, it does a body good: Effects of message framing and motivational orientation on young women's calcium consumption. Journal of Health Psychology. 2013; 18:1296-1306.10.1177/1359105312463587 [PubMed: 23180876]

Gerend MA, Shepherd JE. Using message framing to promote acceptance of the human papillomavirus vaccine. Health Psychology. 2007; 26:745-752.10.1037/0278-6133.26.6.745 [PubMed: 18020847]

Hagger MS, Chatzisarantis NL, Biddle SJ. A meta-analytic review of the theories of reasoned action and planned behavior in physical activity: Predictive validity and the contribution of additional variables. Journal of Sport \& Exercise Psychology. 2002; 24:3-32.

Halvari AEM, Halvari H. Motivational predictors of change in oral health: An experimental test of self-determination theory. Motivation and Emotion. 2006; 30:294-305.10.1007/ s11031-006-9035-8

Higgins ET. Beyond pleasure and pain. American Psychologist. 1997; 52:12801300.10.1037/0003-066X.52.12.1280 [PubMed: 9414606]

Hull SJ. Perceived risk as a moderator of the effectiveness of framed HIV-test promotion messages among women: A randomized controlled trial. Health Psychology. 2012; 31:114-121.10.1037/ a0024702 [PubMed: 21767018]

Jorm AF, Christensen H, Henderson AH, Jacomb PA, Korten AE, Rodgers B. Using the BIS/BAS scales to measure behavioural inhibition and behavioural activation: Factor structure, validity and norms in a large community sample. Personality and Individual Differences. 1998; 26:49_ 58.10.1016/S0191-8869(98)00143-3

Latimer AE, Rivers SE, Rench TA, Katulak NA, Hicks A, Hodorowski JK, Higgins ET, Salovey P. A field experiment testing the utility of regulatory fit messages for promoting physical activity. Journal of Experimental Social Psychology. 2008; 44:826-832.10.1016/j.jesp.2007.07.013 [PubMed: 19543443]

Latimer AE, Williams-Piehota P, Katulak NA, Cox A, Mowad L, Higgins ET, Salovey P. Promoting fruit and vegetable intake through messages tailored to individual differences in regulatory focus. Annals of Behavioral Medicine. 2008; 35:363-369.10.1007/s12160-008-9039-6 [PubMed: 18670833] 
Lee AY, Aaker JL. Bringing the frame into focus: The influence of regulatory fit on processing fluency and persuasion. Journal of Personality and Social Psychology. 2004; 86:205218.10.1037/0022-3514.86.2.205 [PubMed: 14769079]

Liang KY, Zeger SL. Longitudinal data analysis using generalized linear models. Biometrika. 1986; 73:13-22.10.1093/biomet/73.1.13

Loos BG, Craandijk J, Hoek FJ, Wertheim-van Dillen PM, van der Velden U. Elevation of systemic markers related to cardiovascular diseases in the peripheral blood of periodontitis patients. Journal of Periodontology. 2000; 71:1528-1534.10.1902/jop.2000.71.10.1528 [PubMed: 11063384]

Mann T, Sherman D, Updegraff JA. Dispositional motivations and message framing: A test of the congruency hypothesis in college students. Health Psychology. 2004; 23:330334.10.1037/0278-6133.23.3.330 [PubMed: 15099176]

McCaul K, Glasgow R, Gustafson C. Predicting levels of preventive dental behaviors. Journal of the American Dental Association. 1985; 111:601-605. [PubMed: 3863862]

Moher D, Schulz KF, Altman DG. The CONSORT statement: Revised recommendations for improving the quality of reports of parallel-group randomised trials. The Lancet. 2001; 357:11911194.10.1016/S0140-6736(00)04337-3

Nan X. Communicating to young adults about HPV vaccination: Consideration of message framing, motivation, and gender. Health Communication. 2012; 27:10-18.10.1080/10410236.2011.567447 [PubMed: 22276999]

Pakpour AH, Yekaninejad MS, Sniehotta FF, Updegraff JA, Dombrowski SU. The effectiveness of gain- versus loss-framed health messages in improving oral health in Iranian secondary schools: A cluster-randomized controlled trial. Annals of Behavioral Medicine. (in press).

Renner B, Knoll N, Schwarzer R. Age and body make a difference in optimistic health beliefs and nutrition behaviors. International Journal of Behavioral Medicine. 2000; 7:143-159.10.1207/ S15327558IJBM0702_4

Rothman, AJ.; Updegraff, JA. Specifying when and how gain-and loss-framed messages motivate healthy behavior: An integrated approach. In: Keren, G., editor. Perspectives on framing. London, UK: Psychology Press/Taylor \& Francis; 2010. p. 257-278.

Rothman, AJ.; Wlaschin, J.; Bartels, RD.; Latimer, AE.; Salovey, P. How persons and situations regulate message framing effects: The study of health behavior. In: Elliot, AJ., editor. Handbook of approach and avoidance motivation. Mahwah, NJ: Erlbaum; 2008. p. 475-486.

Sallis JF, Prochaska JJ, Taylor WC. A review of correlates of physical activity of children and adolescents. Medicine and Science in Sports and Exercise. 2000; 32:963975.10.1097/00005768-200005000-00014 [PubMed: 10795788]

Sheeran P. Intention-Behavior relations: A conceptual and empirical review. European Review of Social Psychology. 2002; 12:1-36.10.1080/14792772143000003

Sheeran P, Orbell S. Do intentions predict condom use? Meta-analysis and examination of six moderator variables. British Journal of Social Psychology. 1998; 37:231-250.10.1111/j. 2044-8309.1998.tb01167.x [PubMed: 9639864]

Sherman DK, Brick C, Binning KR, Mintzer RE, McCully SE, Updegraff JA. Culture and health communication: The role of cultural exposure in health message framing. 2014 Manuscript in preparation.

Sherman DK, Mann T, Updegraff JA. Approach/avoidance motivation, message framing, and health behavior: Understanding the congruency effect. Motivation and Emotion. 2006; 30:164168.10.1007/s11031-006-9001-5

Sherman DK, Updegraff JA, Mann T. Improving oral health behavior: A social psychological approach. Journal of the American Dental Association. 2008; 139:1382-1387. [PubMed: 18832274]

Stewart J, Jacobs-Schoen M, Padilla M, Maeder L, Wolfe G, Hartz G. The effect of a cognitive behavioral intervention on oral hygiene. Journal of Clinical Periodontology. 1991; 18:219_ 222.10.1111/j.1600-051X.1991.tb00418.x [PubMed: 1856301]

Tedesco L, Keffer M, Davis EL, Christersson LA. Effect of a social cognitive intervention on oral health status, behavior reports, and cognitions. Journal of Periodontology. 1992; 63:567575.10.1902/jop.1992.63.7.567 [PubMed: 1507036] 
Ulleberg P, Rundmo T. Personality, attitudes and risk perception as predictors of risky driving behaviour among young drivers. Safety Science. 2003; 41:427-443.10.1016/ S0925-7535(01)00077-7

Updegraff JA, Sherman DK, Luyster FS, Mann TL. The effects of message quality and congruency on perceptions of tailored health communications. Journal of Experimental Social Psychology. 2007; 43:249-257.10.1016/j.jesp.2006.01.007 [PubMed: 18958299]

Uskul AK, Sherman DK, Fitzgibbon J. The cultural congruency effect: Culture, regulatory focus, and the effectiveness of gain- vs. loss-framed health messages. Journal of Experimental Social Psychology. 2009; 45:535-541.10.1016/j.jesp.2008.12.005

Watt RG, Marinho VC. Does oral health promotion improve oral hygiene and gingival health? Periodontology. 2005; 37:35-47.10.1111/j.1600-0757.2004.03796.x

Weinstein ND. Why it won't happen to me: Perceptions of risk factors and susceptibility. Health Psychology. 1984; 3:431-457.10.1037/0278-6133.3.5.431 [PubMed: 6536498]

Zhao G, Pechmann C. The impact of regulatory focus on adolescents' response to antismoking advertising campaigns. Journal of Marketing Research. 2007; 44:671-687.10.1509/jmkr.44.4.671 


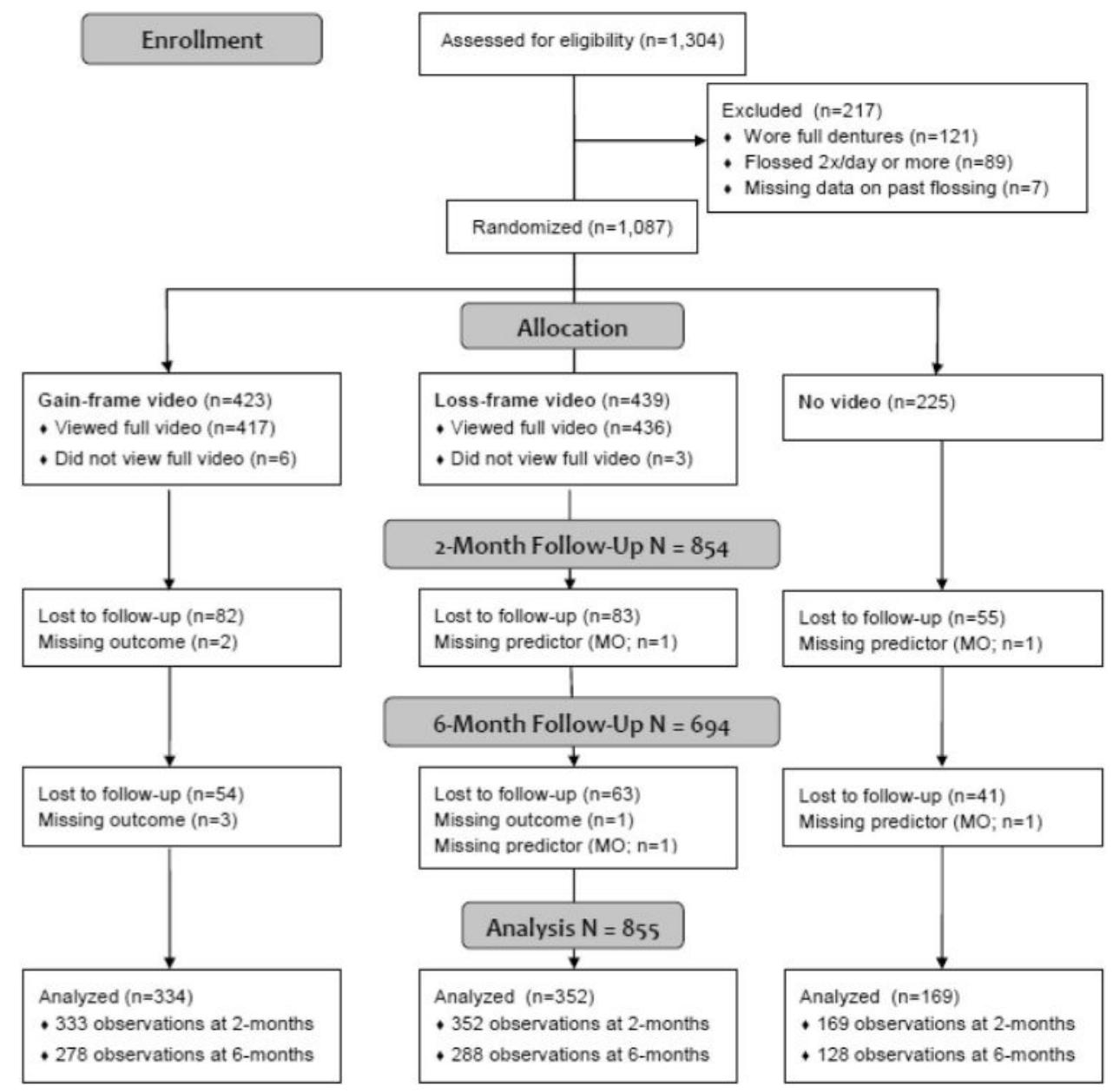

Figure 1.

CONSORT diagram of eligibility, allocation, and attrition. (Note: One participant was missing outcome data at 2 months but remained in the study at 6 months; hence the difference in the 2-month and final analysis $N$ ). 


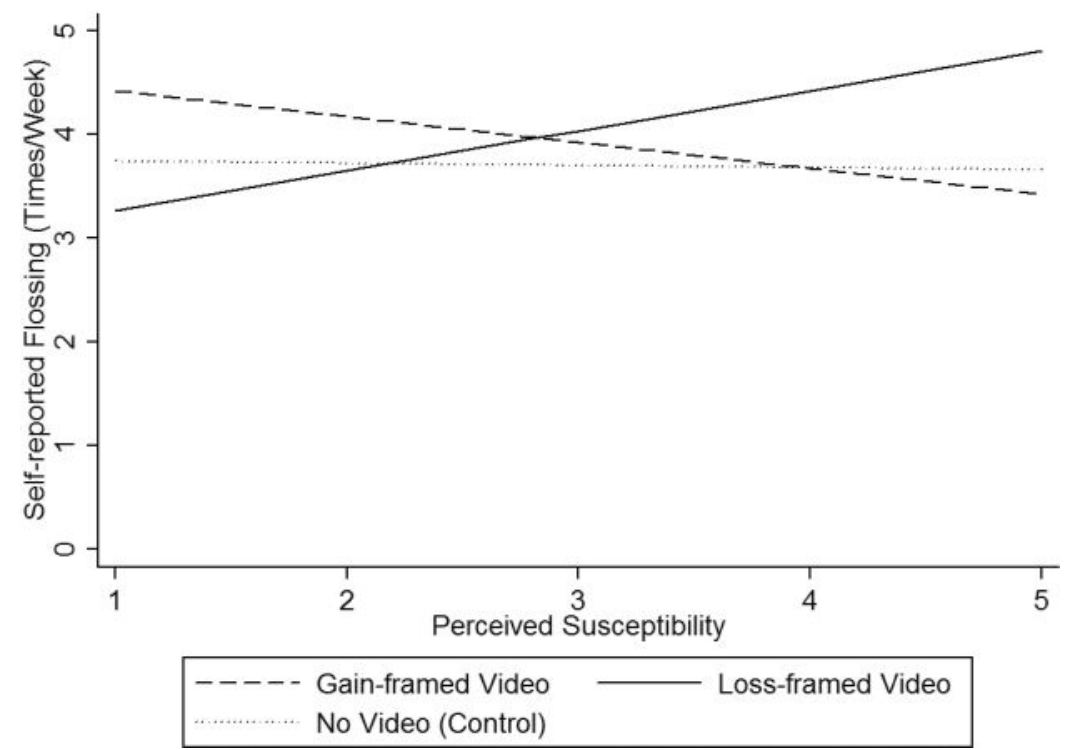

Figure 2.

Interaction between perceived susceptibility and message frame on self-reported flossing (times/week) at the 6-month follow-up. 


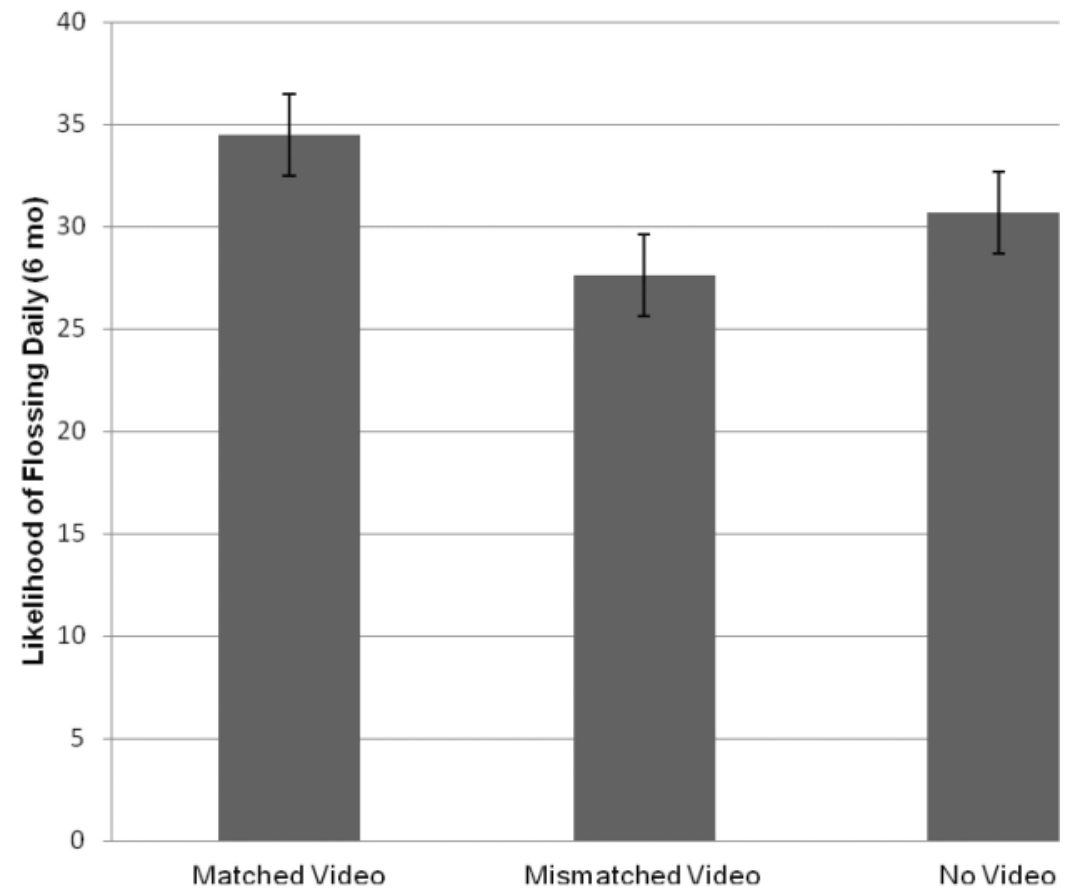

Figure 3.

Predicted likelihood of daily flossing at the 6-month follow-up, depending on whether participant received video matched or mismatched to perceived susceptibility, or no video. Bars represent standard errors of estimates. Planned contrasts show matched video to increase likelihood of daily flossing relative to mismatched and no video combined, and mismatched video to be no different than no video. 


\section{Table 1}

Demographic and Analytical Variable by Condition

\begin{tabular}{|c|c|c|c|}
\hline Age & Gain video $(N=334) 43.69(14.81)$ & Loss video $(N=352) 45.46(15.80)$ & No video $(N=169) 44.25(15.84)$ \\
\hline Female & $46 \%$ & $46 \%$ & $49 \%$ \\
\hline Race: White & $23 \%$ & $22 \%$ & $21 \%$ \\
\hline Race: Black & $25 \%$ & $27 \%$ & $30 \%$ \\
\hline Race: Hispanic & $29 \%$ & $29 \%$ & $27 \%$ \\
\hline Race: Asian & $23 \%$ & $22 \%$ & $22 \%$ \\
\hline Median income & $\$ 47,100$ & $\$ 47,500$ & $\$ 47,900$ \\
\hline Susceptibility & $2.35(.97)$ & $2.39(1.08)$ & $2.50(1.11)$ \\
\hline Motivational orientation & $.08(.57)$ & $.07(.58)$ & $.09(.58)$ \\
\hline Flossing at baseline ${ }^{*}$ & $2.97(2.79)$ & $3.03(2.84)$ & $3.06(2.91)$ \\
\hline Flossing at 2 months & $3.68(3.33)$ & $3.79(3.64)$ & $3.47(3.46)$ \\
\hline Flossing at 6 months & $4.02(3.69)$ & $3.86(3.85)$ & $3.68(3.77)$ \\
\hline Dental visit by 6 months ${ }^{* *}$ & $47 \%$ & $54 \%$ & $48 \%$ \\
\hline
\end{tabular}

Health Psychol. Author manuscript; available in PMC 2015 December 26. 


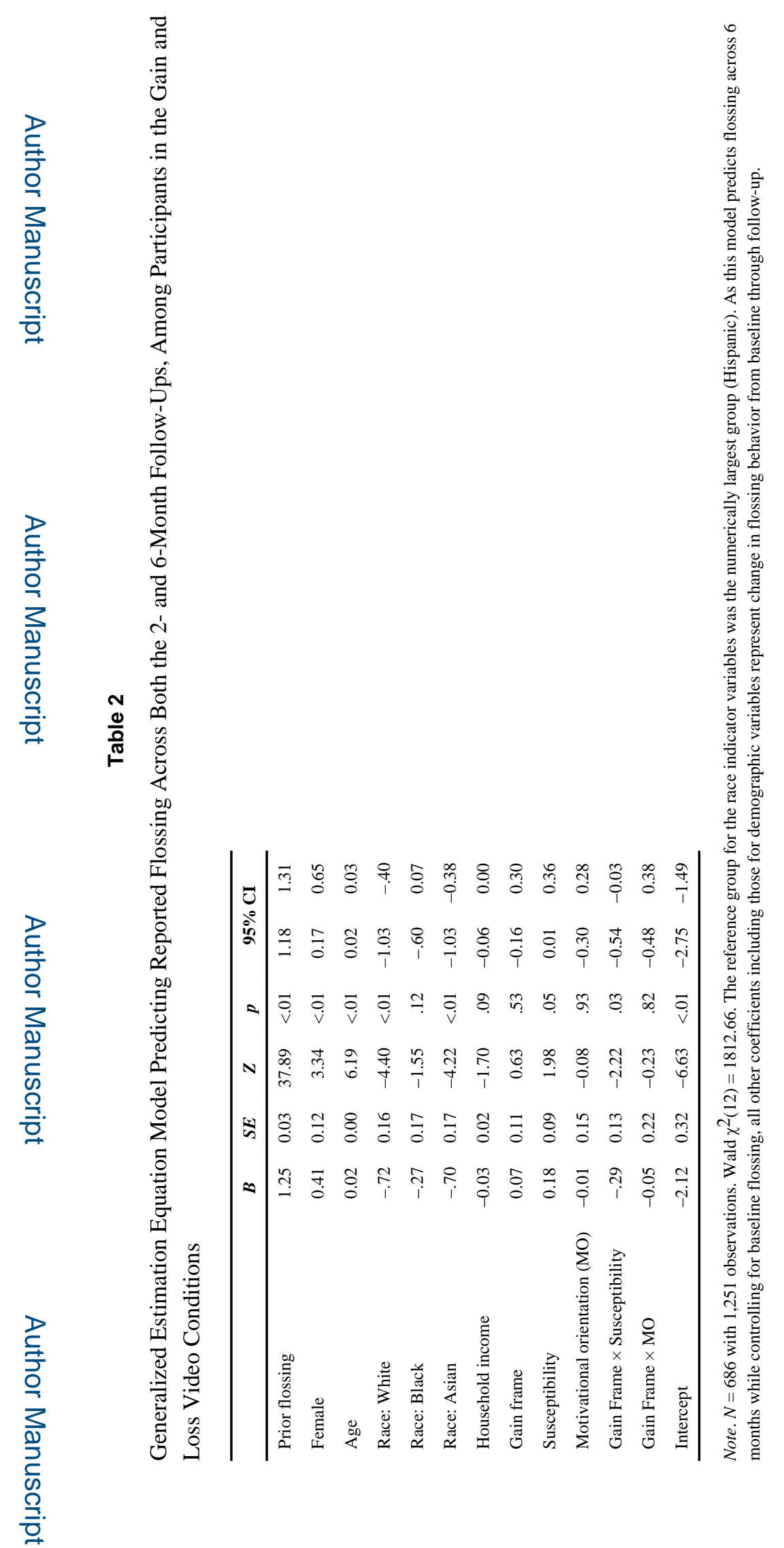

Health Psychol. Author manuscript; available in PMC 2015 December 26. 